\title{
The involvement of $T$ regulatory lymphocytes in a cohort of lupus nephritis patients: a pilot study
}

\author{
Antonella Afeltra • Antonietta Gigante - Domenico Paolo Emanuele Margiotta • \\ Chiara Taffon • Rosario Cianci - Biagio Barbano • Marta Liberatori • \\ Antonio Amoroso • Filippo Rossi Fanelli
}

Received: 23 July 2014/ Accepted: 30 January 2015 / Published online: 27 February 2015

(C) SIMI 2015

\begin{abstract}
T regulator lymphocytes (Tregs) play a key role in the maintenance of immune tolerance and in the development of autoimmune diseases. Expression of Foxp3 is specific for Tregs, and can be used for the identification of these cells. This study investigated the variations of Tregs Foxp3+ in the kidney biopsies inflammatory infiltrate of different lupus nephritis classes compared to that of ANCA glomerulonephritis, acute tubulointerstitial nephritis and nephroangiosclerosis. Sections of paraffin-embedded tissue have been stained by immunohistochemistry with anti-CD3 and anti-FoxP3 antibodies. We find that the ratio of FoxP3+/CD3 + cells is significantly lower in patients with lupus nephritis class IV and in patients with vasculitides than in the course of nephroangiosclerosis, tubulointerstitial nephritis and lupus nephritis class V. The data presented herein demonstrate a decrease of FoxP3+ Treg cells in the inflammatory infiltrate of lupus nephritis, particularly during the most active phases of lupus nephritis, as observed in the course of a IV class nephritis.
\end{abstract}

A. Afeltra - D. P. E. Margiotta

Clinical Medicine and Rheumatology, Integrated Research

Center, Campus Bio-Medico University, Rome, Italy

A. Gigante $(\varangle) \cdot$ M. Liberatori · A. Amoroso - F. Rossi Fanelli Department of Clinical Medicine, Sapienza, University of Rome, Viale dell'Università, 3700185 Rome, Italy

e-mail: Antonietta_gigante@yahoo.it

C. Taffon

Pathology, Integrated Research Center, Campus Bio-Medico University, Rome, Italy

R. Cianci · B. Barbano

Department of Clinical Medicine, Nephrology Unit, Sapienza, University of Rome, Rome, Italy
Keywords T reg lymphocytes - FoxP3 - Lupus nephritis · SLE · Glomerulonephritis

\section{Introduction}

Lupus nephritis is a common and severe complication of systemic lupus erythematosus (SLE). A number of patients have nephritis as a presenting feature that in its severe form can quickly lead to end-stage renal disease or death $[1,2]$. Clinical features of patients with lupus nephritis $(\mathrm{LN})$ range from asymptomatic urinary microhematuria to macroscopic hematuria, or proteinuria; from mild to nephrotic levels, or reduced renal function with rapidly progressive renal failure and hypertension. Renal biopsy shows histological findings that demonstrate a spectrum of vascular, glomerular and tubulointerstitial lesions [2]. LN pathogenesis is attributable mostly to the glomerular deposition of immune complexes and overproduction of $\mathrm{T}$ helper cytokines. Immune complexes deposits promote the inflammatory response by activation of adhesion molecules on endothelium, resulting in the recruitment of pro-inflammatory leukocytes and development of autoimmune injury. Moreover, activated and damaged glomerular cells, infiltrating macrophages, B and $\mathrm{T}$ cells produce cytokines that play a pivotal role as inflammatory mediators to extend renal injury [3-5].

Several potential mechanisms have been reported, involving alterations in $\mathrm{T}$ cell receptor and $\mathrm{B}$ cell receptor expressions, postreceptor downstream signaling, which favor activation of lupus $\mathrm{T}$ and $\mathrm{B}$ cells. Tyrosine-protein kinase (Syk) is recruited at phosphorylated tyrosines on immunoreceptors, including the B cell receptor (BCR), $\mathrm{T}$ cell receptor (TCR) The FcR $\gamma$ chain/Syk complex populates lipid rafts, which are pre-clustered in SLE T cells, and contribute to the hyperexcitable $\mathrm{T}$ cell phenotype found in 
SLE. A series of Janus Kinase/Signal Transducer and Activator of Transcription (Jak-STAT) signaling cytokines, especially type I IFNs, IL-10 and IL-6, as well as the hormone prolactin, have been implicated in the pathogenesis of SLE. Bruton's tyrosine kinase (BTK) is a cytoplasmic enzyme that is indispensable for signaling through the B cell receptor. Oral BTK inhibitor improves disease in lupus prone mice. SLE T cells express increased amounts of nuclear CaMKIV (a serine/threonine kinase that is activated by calcium and then translocates to the nucleus,) which activates CREM $\alpha$ that binds to interleukin IL-2 promoter suppressing the transcription of the IL-2 gene. ROCK, a serine/threonine kinase, which acts downstream of the small GTPase and RhoA, and regulates cytoskeletal dynamics, has been implicated in SLE pathogenesis by phosphorylating ezrin/radixin/moiesin (ERM) and IRF4. There is a growing body of evidence that implicates the PI3K pathway in SLE pathogenesis. Rai, a Shc adapter family member, acts as a negative regulator of antigen receptor signaling in $\mathrm{T}$ and $\mathrm{B}$ cells. RAI (-/-) mice develop lupus like disease with T CD4+ cells polarization to Th17 lymphocytes [6, 7].

Over the past decade, the discovery of new lymphocytes subtypes revolutionized SLE pathogenesis conception. In particular, starting from the late 1990s, T lymphocytes with regulatory properties ( $\mathrm{T}$ regulatory cells or Tregs) have been described. It has been shown that Tregs are able to inhibit $\mathrm{T}$ cell proliferation and cytokine production, and play a critical role in preventing autoimmunity. Tregs play a central role in the regulation of immune responses to selfantigens, allergens, and commensal microbiota as well as immune responses to infectious agents and tumors. Recent studies suggest that human Treg cells are functionally and phenotypically diverse. The most studied Tregs subpopulation express specific marker FoxP3 (Forkhead box $\mathrm{P} 3$ ) which is a protein localized intracellularly that is crucial for regulatory functions. The complete immunophenotype of these cells is CD4+, CD25, lowFoxP3+ [8-12]. Tregs involvement in kidney diseases has been studied mostly in oncology. Studies demonstrate a positive correlation between Tregs infiltration in kidney specimens and prognosis. Moreover, Tregs kidney infiltration may be a prognostic marker of long-term graft function and acute rejection [13, 14]. A number of studies describe Tregs involvement in SLE pathogenesis. Nevertheless, Tregs' role in SLE nephritis remains unknown [15-17].

\section{Materials and methods}

Patients and controls

The study was carried out on renal biopsy samples of 29 patients with histologically proven LN, 5 patients with
ANCA-related glomerulonephritis (ANCA-CrGN), 6 patients with acute tubulointerstitial nephritis (ATIN), and 5 patients with nephroangiosclerosis (NAS). Twenty-nine Caucasian patients with histologically proven LN classified according to the ISN/RPS criteria [18] (25 females, 4 males) were enrolled in the study (class III: 5 patients, class IV: 17 patients, class V: 7 patients). SLE diagnosis was established according to the 1997 revised American Rheumatism Association criteria [19]. SLE disease activity was evaluated using SLEDAI score [20]. The clinical presentation, $24 \mathrm{~h}$ proteinuria, urinalysis, serum creatinine level, and autoimmunity antibodies were recorded at the time of biopsy. Of 29 SLE patients, 1 presented neuropsychiatric involvement, 17 had arthralgia, 6 suffered from serositis and oral ulcers, 7 photosensitivity, 13 presented skin rashes and 11 cytopenia. None of the patients had ever received cyclophosphamide, mycophenolate or rituximab. Other immunosuppressive drugs were suspended for at least 6 months prior to this study except for steroid therapy that was maintained at a dose of no more than $12.5 \mathrm{mg}$ die. At the time of renal biopsy, patients' features were:

- LN: mean age $35.8 \pm 9.3 ; 25$ female, 4 male; serum creatinine $1.4 \mathrm{mg} / \mathrm{dl} \pm 1.3$; mean proteinuria $3.3 \mathrm{~g} /$ $24 \mathrm{~h} \pm 1.8$; SLEDAI score with a score $\geq 6$. All patients had microscopic hematuria.

- ATIN: mean age $56 \pm 18.9 ; 4$ female, 2 male serum creatinine $3.7 \mathrm{mg} / \mathrm{dl} \pm 2.2$; mean proteinuria $0.6 \mathrm{~g} /$ $24 \mathrm{~h} \pm 0.4 ; 50 \%$ of patients had microscopic hematuria.

- ANCA-CrGN: mean age $57 \pm 17.2 ; 3$ female, 2 male serum creatinine $5.1 \mathrm{mg} / \mathrm{dl} \pm 2.5$; mean proteinuria $2.6 \mathrm{~g} / 24 \mathrm{~h} \pm 0.7$. All patients had microscopic hematuria.

- NAS: mean age $54 \pm 11 ; 2$ female, 3 male serum creatinine $0.8 \mathrm{mg} / \mathrm{dl} \pm 0.1$; mean proteinuria $0.8 \mathrm{~g} /$ $24 \mathrm{~h} \pm 0.2$. All patients had microscopic hematuria.

Autoimmunity and autoantibodies

ANA

We used ANA-immunofluorescence assay (ANA-IFA) in which slides prepared from human epithelioid cells (HEp2 cells) as a substrate are incubated with diluted serum. The presence of autoantibodies is detected by fluorescent anti-immunoglobulin antibody, and characteristic morphologic patterns of fluorescent staining are observed. Tests were performed using commercial kits by Biomedis srl (Rome, Italy) according to the manufacturer's instructions. 


\section{Anti-dsDNA}

We used indirect immunofluorescence assay on Chritidia Luciliae substrate incubate with diluted serum. Tests were performed using commercial kits by Biomedis srl (Rome, Italy) according to the manufacturer's instructions.

\section{Anti-cardiolipin antibodies $\operatorname{Ig} G$ and $\operatorname{IgM}$ and anti- beta2GPI}

We used EIA commercial kit by DASIT srl (Milano, Italy) according to the manufacturer's instructions.

\section{Anti-neutrophil cytoplasmic antibodies}

We used commercial immunofluorescence (IF) on ethanolfixed neutrophils kit (Biomedis Srl, Roma) according to the manufacturer's instructions.

Histology and morphometric analysis

Renal tissue was obtained by percutaneous needle biopsy. Tissue cylinders were received within $15 \mathrm{~min}$ from the time of biopsy; specimens from each biopsy were divided into three portions. Two were processed for routine light and electron microscopic examination; the third fragment was embedded in OCT and snap-frozen in liquid nitrogencooled isopentane. 3 to 4 sections from paraffin-embedded blocks were routinely stained with hematoxylin-eosin, PAS and Periodic acid-silver methenamine (PASM) 5 sections from OCT-embedded tissues were cut in a cryostat, brought to phosphate buffered saline PBS, and stained with FITC-conjugated antibodies against human IgA, IgG, IgM, C3c, C1q, Fibrinogen and Kappa and Lambda light chains (Dako Corporation, Glostrup, Denmark). The severity of glomerular, tubulointerstitial and vascular lesions was evaluated according to the standard histological classification of LN, and the corresponding histological class was assigned to each case [18].

Immunohistochemistry was then performed by the streptavidin-biotin method onto additional sections obtained from the paraffin-embedded tissues, using the following primary antibodies:

1. Monoclonal antibody against FoxP3 (Abcam Inc, Cambridge, UK).

2. Monoclonal antibody against CD3 (Dako Corp., Glostrup, Denmark).

Adjacent sections were cut from each block, and collected on two slides; immunostaining for each of the two antibodies was therefore performed in each case. Briefly, endogenous peroxidase was blocked by incubation with
$3 \%$ hydrogen peroxide, and primary antibodies were used at a concentration of $25 \mu \mathrm{g} / \mathrm{mL}$. Sections were then incubated with LSAB2 (Dakocytomation) 3-3'-diaminobenzidine (DAB) was used for color development, and haematoxylin was used for counterstaining. Negative controls were obtained by omitting primary antibodies.

Digital images from the immunostained slides were obtained using ScanScope Digital Slide Scanner (Aperio, Vista, CA, USA) at a $40 \times$ magnification. Quality control of the scanned images and all further analysis were performed using ImageScope software V10.2.1.2315 (Aperio). Slides were analyzed by using the Positive Pixel Count Algorithm $\mathrm{v} 9$, which counts pixels of predetermined color, intensity and saturation; each slide was separately analyzed using the default set of parameters. An algorithm output provided a number of $1+, 2+$ and $3+$ intensity positive pixels, and the number of total pixels in each annotated layer. The total number of pixels consisted of positive and negative pixels excluding the white area of the virtual slides (i.e., the center of tubules, vacuoles, Bowman's capsule space). Since 1+ pixel areas were mostly picking-up weak background staining but not FoxP3 or CD3 immunostaining, only $2+$ and $3+$ areas were regarded as positive for further analysis; the pixel number was then normalized by the whole kidney section area. By this method, it was possible to obtain (1) the number of FoxP3-positive and CD3positive cells in each case, and (2) the ratio of FoxP3positive CD3 cells in each case (Figs. 1, 2).

The study was approved by the Local Ethics Committee Directives of Clinical Medicine Department of Sapienza University of Rome.

\section{Results}

Demographics

The demographic parameters of the samples, at the time of renal biopsy, are reported in Table 1.

CD3+ lymphocytes expression

In sections examined, the number of $\mathrm{CD} 3+$ lymphocytes was significantly higher in patients with acute tubulointerstitial nephritis (ATIN) $\left(5713 / \mathrm{mm}^{2}\right)$ and ANCA crescentic glomerulonephritis $(\mathrm{CrGN})(5121 / \mathrm{mm} 2$,) compared to patients with LN class IV (3558/mm2), LN class III $\left(2491 / \mathrm{mm}^{2}\right)$, nephroangiosclerosis (NAS) $\left(2379 / \mathrm{mm}^{2}\right)$ and $\mathrm{LN}$ class $\mathrm{V}\left(2220 / \mathrm{mm}^{2}\right), p<0.05$.

In particular, we found no statistically significant differences in the expression of CD3 + cells among the three classes of LN. 


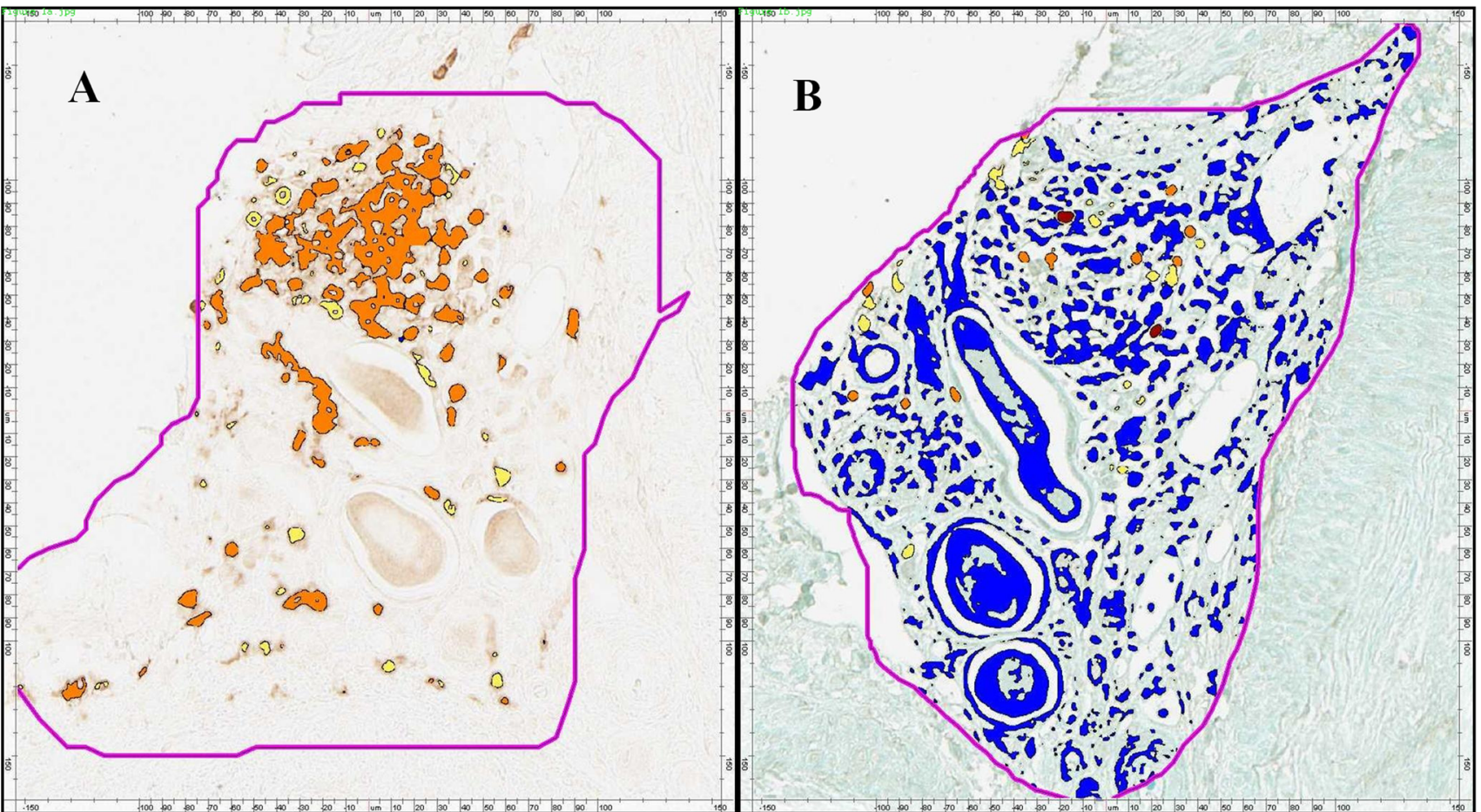

Fig. 1 a CD3 + cells in section of patient with NAS. CD3 infiltration is expressed by a chromatic scale: high-infiltration (brown), mediuminfiltration (orange), low-infiltration (yellow), negative (blue). b FoxP3+ cells in section of patient with NAS. FoxP3 infiltration is expressed by a chromatic scale: high-infiltration (brown), medium-infiltration (orange), low-infiltration (yellow), negative (blue) (color figure online)

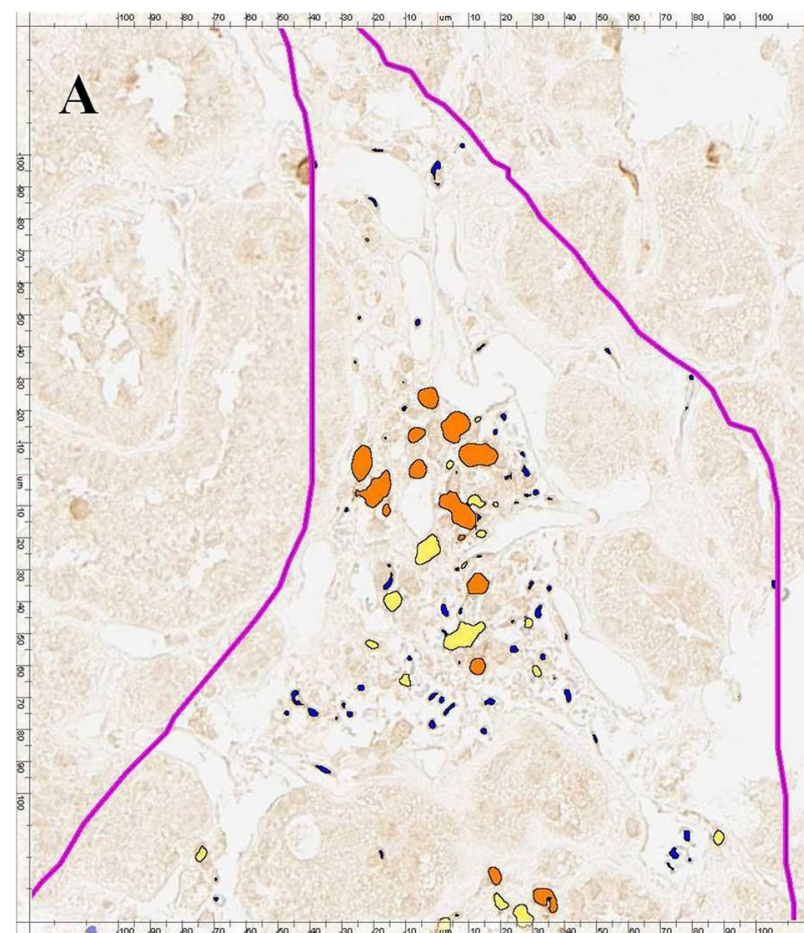

Fig. 2 a $\mathrm{CD} 3+$ cells in section of patient with class IV LN. CD3 infiltration is expressed by a chromatic scale: high-infiltration (brown), medium-infiltration (orange), low-infiltration (yellow), negative (blue). b FoxP3 + cells in section of patient with class IV

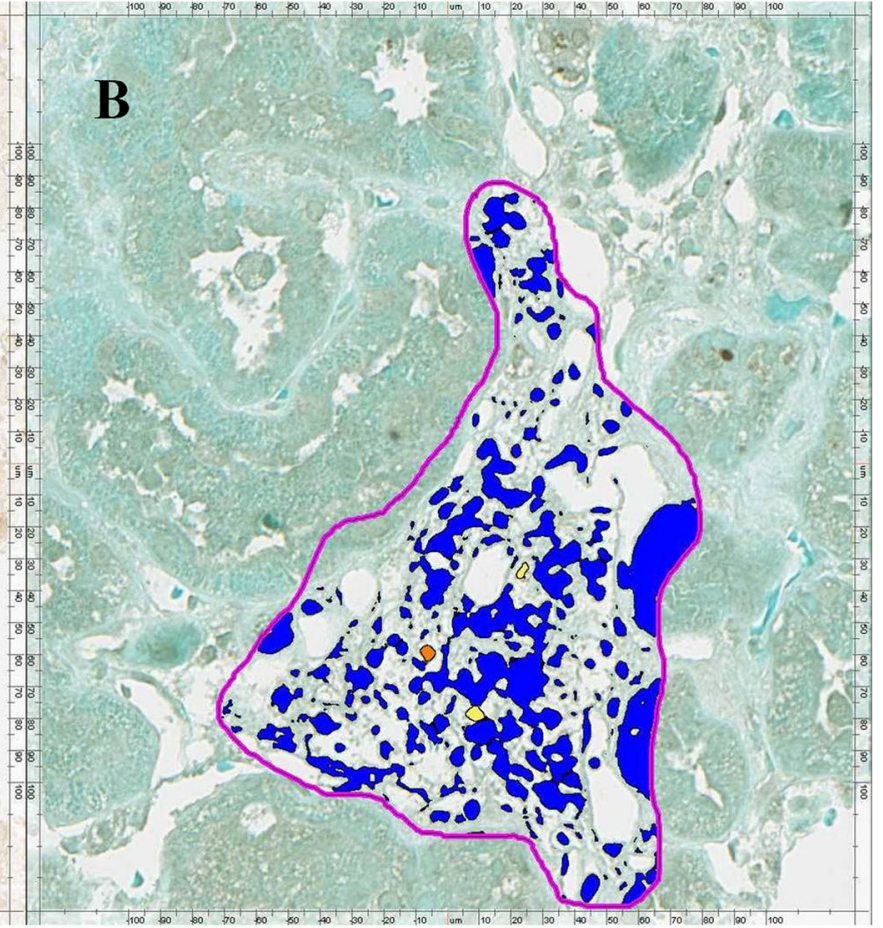

LN. FoxP3 infiltration is expressed by a chromatic scale: highinfiltration (brown), medium-infiltration (orange), low-infiltration (yellow), negative (blue) (color figure online) 
Table 1 The demographic parameters of the samples, at the time of renal biopsy

\begin{tabular}{|c|c|c|c|c|}
\hline & $\mathrm{LN}$ & ANCA-CrGN & ATIN & NAS \\
\hline$N$ & 27 & 3 & 6 & 2 \\
\hline Age (years) $($ mean $\pm \mathrm{SD})$ & $35.8 \pm 9.3$ & $57 \pm 17.2$ & $56 \pm 18.9$ & $54 \pm 11$ \\
\hline Systolic blood pressure $(\mathrm{mmHg})($ mean $\pm \mathrm{SD})$ & $130 \pm 15$ & $140 \pm 16$ & $135 \pm 14$ & $145 \pm 20$ \\
\hline Diastolic blood pressure $(\mathrm{mmHg})($ mean $\pm \mathrm{SD})$ & $85 \pm 5$ & $90 \pm 8$ & $90 \pm 6$ & $90 \pm 8$ \\
\hline BMI $\left(\mathrm{kg} / \mathrm{m}^{2}\right)($ mean $\pm \mathrm{SD})$ & $24.6 \pm 4$ & $23.9 \pm 3$ & $23 \pm 3.5$ & $24.5 \pm 3.6$ \\
\hline Smokers $(\%)$ & 0 & 0 & 0 & 0 \\
\hline Hypertension (ESC criteria) (\%) & 37 & 33 & 50 & 50 \\
\hline Dyslpidemia (ATPIII/NCEP criteria) (\%) & 29.6 & 33 & 33.3 & 50 \\
\hline Type 2 diabetes (ADA criteria) (\%) & 0 & 0 & 0 & 0 \\
\hline CAD history (AHA criteria) (\%) & 0 & 0 & 0 & 0 \\
\hline Disease duration (years) (mean $\pm \mathrm{SD}$ ) & $8.1 \pm 2.3$ & & & \\
\hline SLEDAI pt (mean \pm SD) & $14 \pm 4$ & & & \\
\hline \multicolumn{5}{|l|}{ Therapy (\%) } \\
\hline CCS per os & 44 & & & \\
\hline $\mathrm{CCS}+\mathrm{HCQ}$ & 55.5 & & & \\
\hline $\mathrm{CCS}+\mathrm{CyA}$ & 0 & & & \\
\hline $\mathrm{CCS}+\mathrm{AZA}$ & 0 & & & \\
\hline $\mathrm{CCS}+\mathrm{MTX}$ & 0 & & & \\
\hline $\mathrm{CCS}+\mathrm{MFM}$ & 0 & & & \\
\hline $\mathrm{CCS}+\mathrm{CyC}$ & 0 & & & \\
\hline Rituximab & 0 & & & \\
\hline \multicolumn{5}{|l|}{ Therapy history (\%) } \\
\hline CCS bolus & 3.7 & & & \\
\hline CyC bolus & 0 & & & \\
\hline Rituximab & 0 & & & \\
\hline ANA $\geq 1: 160(\%)$ & 100 & 0 & 0 & 0 \\
\hline Anti-dsDNA (\%) & 55.5 & 0 & 0 & 0 \\
\hline ENA Sm $(\%)$ & 37.3 & 0 & 0 & 0 \\
\hline ANCA $(\%)$ & 0 & 100 & 0 & 0 \\
\hline $\mathrm{LAC}(\%)$ & 0 & 0 & 0 & 0 \\
\hline Anti-phospholipids (\%) & 0 & 0 & 0 & 0 \\
\hline Hematuria $(\%)$ & 100 & 100 & 50 & 100 \\
\hline Proteinuria $24 \mathrm{~h}(\mathrm{~g} / \mathrm{L})($ mean $\pm \mathrm{SD})$ & $3.3 \pm 1.8$ & $2.6 \pm 0.7$ & $0.6 \pm 0.4$ & $0.8 \pm 0.2$ \\
\hline Serum creatinine $(\mathrm{mg} / \mathrm{dl})($ mean $\pm \mathrm{SD})$ & $1.4 \pm 1.3$ & $5.1 \pm 2.5$ & $3.7 \pm 2.2$ & $0.8 \pm 0.1$ \\
\hline
\end{tabular}

FoxP3+ lymphocytes expression

Considering the totality of patients with LN (LN-IV, LN-V, LN-III together) we find a significant reduction in the ratio FoxP3 + cells/CD3 + cells in SLE compared to subjects with ATIN and NAS, $p<0.05$. The difference of the ratio FoxP3 + cells/CD3 + cells between SLE and ANCA-CrGN is not statistically significant.

We observe a statistically significant reduction of the ratio FoxP3+/CD3+ in LN-IV compared to NAS, to ATIN and to LN-V; $p<0.05$ for LN-IV vs NAS, ATIN, LN-V; $p=\mathrm{ns}$ for LN-III and LN-IV vs ANCA-CrGN.

\section{Discussion}

SLE pathogenesis is a complex process involving both innate and adaptive immunity, and both humoral and cellular immune compartments. Literature data, derived from in vitro studies, works on mouse models and human research, demonstrate the centrality of lymphocyte dysregulation in SLE pathogenesis. In recent years, to the duality $\mathrm{T}$ lymphocytes-B lymphocytes, in part focused on Th1/Th2 paradigm, were added new lymphocyte mediators, leading to a revolution in the concepts of the autoimmune diseases pathogenesis $[21,22]$. The discovery of 
Foxp3 as a specific intracellular marker of $\mathrm{T}$ lymphocytes with regulatory function (Tregs) gave, over the past decade, a new push to research on immuno-regulation [23]. Tregs, and in particular the Tregs with phenotype CD4+ $\mathrm{CD} 25$ high+ FoxP3+, play a crucial role in maintaining immune homeostasis, ensuring tolerance towards selfantigens, and modulating inflammatory responses towards exogenous antigens and to allergens [24]. LN is one of the most serious manifestations of SLE. Recent evidence suggests a possible involvement of Tregs in LN. In a study of human LN, levels of circulating Tregs and the expression of serum and urinary TGF- $\beta 1$ were assessed. This study demonstrates a significant reduction in the frequency of CD4+ CD25 high and CD4+ CD25+ FoxP3+ with respect to the total $\mathrm{CD} 4+\mathrm{T}$ lymphocytes in patients with LN. The decreased Tregs count is accompanied by low serum levels and increased urinary levels of TGF $\beta 1$. In NZB/NZWF1 mice, the depletion of CD4+ CD25+ accelerates the development of membrano-proliferative glomerulonephritis. The adoptive transfer of CD4+ $\mathrm{CD} 25+$ in knock-out mice for these lymphocytes retards the development of proliferative glomerular lesions [12, 15-17, 25]. To date, this is the first study targeted to analyze the Tregs expression in the inflammatory infiltrate of LN renal biopsies, using FoxP3 as Tregs marker. In order to assess the comparative expression of FoxP3 in SLE patients we used subjects with different type of kidney disease: ANCA-CrGN, ATIN and NAS as a control sample. In addition, we evaluated the differences in FoxP3 expression in the context of different LN classes. The use of a heterogeneous control group has allowed us to compare the patients with SLE in three different conditions: nephropathy based on chronic inflammatory status as ANCA-CrGN, nephropathy based on toxic or infectious trigger, and nephropathy based on degenerative process. In line with the literature data regarding the magnitude of the inflammatory infiltrate in nephritis, we find a high expression of $\mathrm{CD} 3+$ cells in patients with ATIN, and to a lesser extent in patients with ANCA-CrGN, compared to patients with LN and NAS. In fact, ATIN, caused by toxic or infectious agents, and renal vasculitis are characterized by an abundant inflammatory infiltrate. Among the LN classes, the patients with class IV have a greater inflammatory infiltrate than the class V and class III. In fact, in the class IV, the development of glomerulo-proliferative lesions is supported by a significant inflammatory stimulation $[18,26]$. Tregs play an important role in the maintenance of immune tolerance to self, and inhibit the development of SLE [27]. FoxP3+ Tregs are significantly decreased, accompanied by increased in Th17 cells in LN patients, suggests that Th17/Treg functional imbalance may be involved in the pathogenesis of renal damage in SLE patients [28]. In lupus nephritis biopsy, we find reduced FoxP3 + Tregs in relation to the total number of lymphocytes of the inflammatory infiltrate (expressed as a ratio cells of FoxP3+/CD3+) Moreover, in our paper we describe a minimal FoxP3+ Tregs expression in class IV lupus nephritis. We describe only quantitative data, and cannot analyze the inhibitory properties of FoxP3 + Tregs. However, we speculate that a numerical hypo-expression of FoxP3+ Tregs in class IV lupus nephritis may take part in the immunological dysregulation leading to a glomeruloproliferative pattern typical of class IV lupus nephritis.

Considering overall $\mathrm{LN}$ patients, we find a reduced Tregs number in relation to total lymphocytes of the inflammatory infiltrate (expressed as a ratio cells of FoxP3+/ $\mathrm{CD} 3+$ ) compared to ATIN and NAS. We find no differences between LN and ANCA-related glomerulonephritis. These findings might suggest that a deficiency of Tregs may facilitate inflammatory glomerular damage based on autoimmune process (such as LN) and this might be irrelevant in inflammatory conditions based on infectious or toxic trigger. To further confirm this speculation, our data show that the Tregs expression is minimal in LN class IV compared to LN class III and V. In fact, the LN class IV is characterized by an inflammatory infiltrate more intense and active than other classes. According to literature data, it is possible to speculate about a role of FoxP3+ Tregs in autoimmune nephropathies, and in particular, in LN. A deficit of FoxP3+ Tregs may facilitate the processes of organ damage on an autoimmune basis, and be crucial in determining proliferative glomerular lesions, as seen in $\mathrm{LN}$ class IV. It should be emphasized that this is a preliminary study. The small sample size does not allow further analysis. The results need to be confirmed and validated in a study with an appropriate simple size and perhaps in a longitudinal study relating the Tregs infiltration with the renal outcome of LN patients.

\section{Conflict of interest None.}

Statement of human and animal rights All procedures performed in studies involving human participants were in accordance with the ethical standards of the institutional and/or national research committee and with the 1964 Helsinki declaration and its later amendments or comparable ethical standards. This article does not contain any studies with animals performed by any of the author.

Informed consent Informed consent was obtained from all individual participants included in the study.

\section{References}

1. Manzi S (2009) Lupus update. Clevel Clin J Med 76(2):137-142

2. Ippolito A, Petri M (2008) An update on mortality in systemic lupus erythematosus. Clin Exp Rheumatol 26(5):S72-S79

3. Sarzi-Puttini P, Atzeni F, Iaccarino L et al (2005) Environment and systemic lupus erythematosus: an overview. Autoimmunity 38(7):465-472 
4. Gigante A, Gasperini ML, Afeltra A et al (2011) Cytokines expression in SLE nephritis. Eur Rev Med Pharmacol Sci 15(1):15-24

5. Mason LJ, Isenberg D (2005) The pathogenesis of systemic lupus erythematosus. In: Davidson AM, Cameron JS, Grunfeld JP et al (eds) Oxford textbook of clinical nephrology. Oxford University Press, Oxford, England, pp 809-829

6. Markopoulou A, Kyttaris VC (2013) Small molecules in the treatment of systemic lupus erythematosus. Clin Immunol 148(3):359-368

7. Savino MT, Ulivieri C, Emmi G et al (2013) The Shc family protein adaptor, Rai, acts as a negative regulator of Th17 and Th1 cell development. J Leukoc Biol 93(4):549-559

8. Wing K, Sakaguchi S (2010) Regulatory T cells exert checks and balances on self tolerance and autoimmunity. Nat Immunol 11(1):7-13

9. Weaver CT, Hatton RD (2009) Interplay between the TH17 and TReg cell lineages: a (co-) evolutionary perspective. Nat Rev Immunol 9(12):883-889

10. Torchinsky MB, Blander JM (2010) T helper 17 cells: discovery, function, and physiological trigger. Cell Mol Life Sci 67(9):1407-1421

11. Bonelli M, Smolen JS, Scheinecker C (2010) Treg and lupus. Ann Rheum Dis 69:65-66

12. La Cava A (2009) The busy life of regulatory $\mathrm{T}$ cells in systemic lupus erythematosus. Discov Med 8(40):13-17

13. Dummer CD, Carpio VN, Gonçalves LF et al (2012) FOXP3+ regulatory $\mathrm{T}$ cells: from suppression of rejection to induction of renal allograft tolerance. Transpl Immunol 26(1):1-10

14. Li XC, Turka LA (2010) An update on regulatory $\mathrm{T}$ cells in transplant tolerance and rejection. Nat Rev Nephrol 6(10):577-583

15. Horwitz DA, Zheng SG, Gray JD (2008) Natural and TGF- $\beta$ induced Foxp3 + CD4+ CD25+ regulatory T cells are not mirror images of each other. Trends Immunol 29(9):429-435

16. Zheng SG, Wang J, Horwitz AD (2008) Cutting edge) Foxp3+ $\mathrm{CD} 4+\mathrm{CD} 25+$ regulatory Tcells induced by IL-2 and TGF- $\beta$ are resistant to Th17 conversion by IL-6. Journal of Immunology 180(11):7112-7116
17. Kaplan JM, Woodworth L, Smith K et al (2008) Therapeutic benefit of treatment with anti- thymocyte globulin and latent TGF- $\beta 1$ in the MRL/lpr lupus mouse model. Lupus 17(9):822-831

18. Weening JJ, D’Agati VD, Schwartz MM et al (2004) The classification of glomerulonephritis in systemic lupus erythematosus revisited. J Am Soc Nephrol 15(2):241-250

19. Hochberg MC (1997) Updating the American College of Rheumatism revised criteria for the classification of systemic lupus erythematosus. Arthr Rheum 40:17-25

20. Bombardier C, Gladman DD, Urowitz MB et al (1992) Derivation of SLEDAI: a disease activity index for lupus patients. Arthr Rheum 35:630-640

21. Hahn BH, Karpouza GA, Tsao BP (2005) Pathogenesis of systemic lupus erythematosus. In: Harris ED (ed) Kelley's textbook of rheumatology, 7th edn. WB Saunders, Philadelphia, pp 1174-1200

22. Rahman A, Isenberg DA (2008) Systemic lupus erythematosus. N Engl J Med 358(9):929-939

23. Shevach EM (2011) Biological functions of regulatory $\mathrm{T}$ cells. Adv Immunol 112:137-176

24. Takakubo Y, Konttinen YT (2012) Immune-regulatory mechanisms in systemic autoimmune and rheumatic diseases. Clin Dev Immunol 2012:941346. doi:10.1155/2012/941346

25. Tucci M, Stucci S, Strippoli S et al (2010) Cytokine overproduction, T-cell activation, and defective t-regulatory functions promote nephritis in systemic lupus erythematosus. J Biomed Biotechnol 2010:457146. doi:10.1155/2010/457146

26. Grande JP, Balow JE (1998) Renal biopsy in lupus nephritis. Lupus 7(9):611-615

27. Wang P, Zheng SG (2013) Regulatory T cells and B cells, implication on autoimmune diseases Int J. Clin Exp Pathol 6:2668-2674

28. Xing Q, Wang B, Su H et al (2012) Elevated Th17 cells are accompanied by FoxP3 + Treg cells decrease in patients with lupus nephritis. Rheumatol Int 32:949-958 